\title{
EDUCACIÓN A LO LARGO DE LA VIDA: AULAS DE MAYORES
}

\author{
LIFE LONG LEARNING: SENIOR CLASSROOMS
}

Pilar Moreno-Crespo ${ }^{12}$

Universidad de Extremadura

\section{RESUMEN}

En las siguientes líneas podemos comprobar cómo ha habido una evolución en el concepto educativo hacia la educación a lo largo de toda la vida. Se amplían, de este modo, los márgenes educativos que se circunscribían a la escuela, invadiendo otros ámbitos de la comunidad, entornos, ámbitos, así como otras etapas de la vida. De este modo se diversifica la oferta formativa a la que pueden acceder las personas adultas atendiendo a la necesidad de educación permanente que posee la sociedad. En el presente artículo trataremos de analizar brevemente las principales modalidades educativas a las que tienen acceso las personas mayores en España. Para finalizar, abordamos el caso de las Aulas de Mayores. Se trata de un tipo de educación exclusiva para personas adultas mayores. Esta modalidad ha tenido una alta aceptación aumentando el número de programas en las últimas décadas. Consiste en una oferta programática que realizan diversas instituciones, principalmente universidades, dirigidas a personas de más de 50/55 años, sin exigencia de titulación previa con la obtención final de un título no competitivo. Se trata de una modalidad de una alta flexibilidad programática. La metodología llevada a cabo es el autoaprendizaje, el cual bebe de las experiencias del alumnado, contando para su puesta en práctica con docentes especialista en la materia. Esta formación es de carácter formal y sociocultural atendiendo las necesidades e inquietudes socioeducativa de sus participantes.

Palabras clave: Educación Permanente, Educación de adultos, Animación cultural, Educación de adultos mayores, Enseñanza Superior

\footnotetext{
12 Este trabajo es resultado de la revisión parcial de la investigación desarrollada para la Tesis Doctoral de la autora (no publicada).
} 


\section{ABSTRACT}

In the following lines we can see how there has been an evolution in the educational concept to Life Long Learning. Expand, thus educational margins that were restricted to school, invading other areas of community, environment, areas, and other stages of life. Thus the training offer that is accessible to adults with the need of continuing education has diversified society. In this article we will briefly analyze the main educational methods that are accessible to older people in Spain. Finally, we address the case of Senior Classrooms. This is a unique kind of education for older adults. This method has a high acceptance by increasing the number of programs in recent decades. It consists of a program offer that perform various institutions, mainly universities, aimed at people over $50 / 55$ years, with no requirement for prior certification with the final production of a non-competitive title. It is a form of high programmatic flexibility. The methodology is carried out self-study, which draws on the experiences of students, counting for its implementation with subject specialist teachers. This training is formal and socio-cultural and socio-educational serving the needs concerns of its participants.

Keywords: Lifelong education, Adult education, Cultural Animation, Seniors Education, Higher education

\section{INTRODUCCIÓN}

Hasta no hace mucho, para referirnos al educando se han utilizado expresiones como el niño o el joven, ya que se identificaba con el espacio físico de la escuela y circunscrito al ámbito de la niñez y de la juventud. La educación permanente es un concepto educativo actual que cambia esta perspectiva y que reconoce el fundamento de una educación para personas adultas que vaya más allá de la educación compensatoria, ya que plantea que el individuo debe mantenerse activo a nivel educativo a lo largo de toda su vida, lo cual conlleva repercusiones en el sistema educativo y en las políticas educativas desarrolladas por las instituciones. La Educación Permanente se esfuerza por dar respuesta a la necesidad de hacer frente al constante cambio en el que nos hallamos y que nos obliga a la actualización permanente o a caer en lo que Pérez Serrano denomina obsolescencia cultural (2001).

Por este motivo, en los sesenta la mirada de la sociedad y de los gobiernos se dirige a la educación, en un tiempo en el que se realizan afirmaciones como que la escuela ha muerto (Reimer) o que hay que tender a una des-educación obligatoria (Goodman). Es un momento en 
el que se replantean los conceptos, llegando a surgir corrientes educativas extremas, entre las que podemos destacar la más radical: el movimiento de la des-escolarización.

En este caldo de cultivo, se genera la situación propicia para diagnosticar la educación y proponer estrategias de mejora que favorezcan la formación de la ciudadanía del futuro. Es en este escenario, donde comienza a reconocerse el valor de la educación permanente (Life Long Learning, LLL) con el propósito de que la persona adquiera destrezas, habilidades, capacidades que le permitan hacer, ser, saber y convivir en sociedad, y que le permitan adaptarse a los cambios constantes del entorno. En este sentido, Sarrate (2002, p. 18-19) reconoce la labor de los organismos intergubernamentales en la enérgica aparición de la expresión "Educación Permanente", aportando que: "Se trata de un nuevo paradigma fundamentado en el principio de que la educación debe estar presente desde el inicio hasta el final del ciclo vital de la persona".

El vertiginoso proceso de avance científico envuelve nuestro día a día con asombrosas investigaciones y descubrimientos, con la agilidad con los que podemos contar con ellos a través de los nuevos cauces de información y comunicación, así como con la necesidad constante de mantenernos actualizados haciendo frente a las nuevas demandas educativas y profesionales. En ocasiones parece que tuviéramos el mundo en nuestras manos y otras que nos desbordara y saturara. Esta situación exige establecer un proceso continuo de modernización con el objeto de adquirir y mantener las herramientas que nos permiten enfrentarnos a la apresurada actualidad cambiante. Se evidencia la necesidad de formación y, en especial, de educación en personas adultas (entre los que se encuentran los adultos mayores).

En los siguientes apartados se definen los conceptos relativos a la educación a lo largo de toda la vida, así como la educación en la adultez. De igual forma, se contempla la educación de adultos desde la formación reglada (Sistema Educativo Español), formal y sociocultural, analizando el ejemplo de las Aulas de Mayores como oferta socioeducativa para personas adultas mayores.

\section{EDUCAR A LO LARGO DE TODA LA VIDA Y EDUCAR EN LA ADULTEZ}

La educación permanente se brinda como recurso imprescindible para el avance de la sociedad. Si seguimos el ejemplo de países como EEUU o Japón, deberemos intensificar las políticas en educación y formación, asociando estas actuaciones al bienestar social y la mejora 
de la competitividad (Ortiz Colón, 2004). En este sentido, Sarrate y Pérez de Guzmán (2005, p. 42), hacen hincapié en la importancia de intensificar la educación permanente cuando destacan los motivos que acompañan la génesis del concepto de educación a lo largo de toda la vida:

\begin{abstract}
Debe tenerse presente que hemos llegado al comienzo de este nuevo siglo con el siguiente escenario: el renacimiento del espíritu del capitalismo clásico y la consiguiente deshumanización, la globalización de la economía mundial, una revolución tecnológica imparable, la aparición de bolsas de miseria en todos los países y las migraciones masivas, que plantean problemas de integración a las sociedades más ricas. Surge, de este modo, la necesidad de una educación en todas las etapas del ciclo vital, que facilite a la persona la formación adecuada para adaptarse, transformar su entorno y conseguir su promoción humana.
\end{abstract}

Sanz Fernández (2002, cit. en Moreno-Crespo, 2011), destaca entre los fenómenos más importantes que influyen en el progreso de la educación permanente y de la formación en la sociedad actual: 1) la necesidad de aprender a emprender, comprendiendo que existe una caducidad de la formación y lo aprendido; 2) Necesidad de simultanear lo global y lo local, lo específico y lo general, descubriendo la realidad poliédrica a la que tenemos acceso desde diversas perspectivas, que nos permite deconstruir el proceso de enseñanza-aprendizaje (espacio, tiempo, método, jerarquía, etc.); 3) La razón parece volver a encontrar su salud, cuestionando el desarrollo puramente instrumental de la razón, buscando espacios de intervención más autónomos y afines de la realidad humana.

Las corrientes actuales, basadas en la educación permanente y en los cuatro pilares de la educación estipulados por Delors en el informe La educación encierra un tesoro, están influenciando los sistemas educativos que inevitablemente tienden a la promoción del protagonismo y responsabilidad del alumnado en su propio aprendizaje, así como a la figura del docente como guía y/o acompañante del proceso educativo (Aguaded y Pérez Rodríguez, 2007; Olmedo Moreno, 2013; Palomares Ruiz, 2011 ).

El reconocimiento de la educación a lo largo de toda la vida desvela que la formación se extiende más allá de los límites infantoescolares, descubriendo un aspecto formativo que ya existía, la educación de personas adultas. En ocasiones se utilizan como términos sinónimos o puede llevar a confusiones entre ambos, por ello consideramos fundamental aclarar que la Educación Permanente abarca todo el ciclo vital y la Educación de Adultos exclusivamente la 
etapa de la adultez (Mayán Santos, 2001), cuestión que destacan Medina Ferrer, Llorent García, Llorent Bedmar (2013, p. 514) al afirmar que: "Ofrecer una definición de educación permanente requiere de una contundente distinción con la educación de adultos, ambas imbricadas en su trayectoria histórica [...]".

Por otro lado, dentro del concepto de Educación de personas adultas se encuentran incluidas las personas adultas mayores, las cuales se localizan en un momento diferente de su vida desde la perspectiva laboral, social, familiar, biológica, económica, espiritual, etc. Refiriéndonos a este colectivo, Cruz Díaz (2013, p. 63) señala que: "En torno a los años 50-60 se plantea la necesidad de abordar nuevas políticas sociales a favor de las personas mayores, tradicionalmente centradas en torno a las pensiones, la asistencia sanitaria y los servicios sociales".

A finales del siglo XX encontramos en España el modelo lineal que contempla un periodo formativo, que abarcaba el periodo inicial de la vida, seguido del ejercicio profesional y finalmente la llegada de la jubilación (Moreno-Crespo y Pérez Pérez, 2014). Por otro lado, un sector de la población no tuvo acceso a una formación básica, principalmente en la primera mitad de dicho siglo, por lo que los índices de analfabetismo siguen siendo una asignatura pendiente entre las personas adultas mayores de nuestra sociedad, junto a los nuevos analfabetismos (Junta de Andalucía, 2010; Gobierno de España, 2011).

Debemos añadir que el avance en número y proporción del grupo poblacional de personas mayores en el último centenar de años ha hecho que las miradas de las instituciones se centren en las necesidades e inquietudes socioeducativas de este colectivo, siendo conscientes de las repercusiones en la calidad de vida derivado de ello, así como en el ahorro en recursos que esto puede llegar a suponer (Prieto Jiménez y Moreno-Crespo, 2009; Moreno-Crespo, 2010; Moreno-Crespo, 2011; Moreno-Crespo, Prieto Jiménez y Amador Muñoz, 2009).

Podemos afirmar que la sociedad, las instituciones y las políticas socioeducativas se encuentran comprometidas con el aprendizaje a todas las edades, ajustando las propuestas formativas a los procesos vitales (García Mínguez, 2004). Aprender a cualquier edad se orienta a producir una adaptación positiva de la persona a cada etapa del ciclo vital en el que se encuentre, para llegar y mantener una mayoría de edad dentro de la sociedad como ciudadano activo y productivo en ésta (Moreno-Crespo y Cruz Díaz, 2012; Moreno-Crespo, Pérez de Guzmán Puya y Rodríguez Díez, 2012). 
Es fundamental que el aprendizaje a lo largo de toda la vida ofrezca respuestas a las nuevas necesidades y oportunidades de cambio que van surgiendo día a día. Entre éstas, Moreno-Crespo (2011) menciona la importancia para el colectivo de personas adultas mayores la adquisición y mantenimiento de hábitos de vida saludables, los cuales repercuten en el desarrollo con calidad de vida del proceso de envejecimiento, así como el afrontamiento de la jubilación y la participación en la sociedad del ocio y tiempo libre.

El análisis de la oferta formativa para personas adultas encontramos tres dimensiones del campo educativo interrelacionados y orientados al desarrollo personal que describimos a continuación según Sarrate (2002; cit. en Moreno-Crespo, 2011, pp. 295-296):

$\checkmark$ Aprendizajes básicos: Conjunto de conocimientos, valores, destrezas y actitudes, indispensables para toda la población, que permiten desenvolverse eficazmente en la vida y servir de soporte para seguir formándose; así como posibilitar el diálogo con la cultura ambiental y participar activamente en la sociedad.

$\checkmark$ Formación para la vida laboral: Comprende la formación profesional inicial, así como la ampliación, actualización y revocación de los conocimientos de índole laboral.

$\checkmark$ Educación para la participación cívico-social y cultural: Capacidad de actuar junto con otros, sin que por ello desaparezca el valor personal de la acción, considerándose como una propiedad de la persona que implica donación y responsabilidad.

En relación a la educación de personas adultas mayores Montoya (2002, p. 209) entiende que la educación permanente en las personas mayores: "[...] debe darse en el mundo de los adultos bien sea como difusión cultural, bien como reciclaje profesional. Las personas mayores pueden acceder a esa educación permanente si no como reciclaje, sí como enriquecimiento cultural".

Tras aclarar los términos educación de personas adultas y educación a lo largo de toda la vida, pasamos a ver en los siguientes apartados diferentes actuaciones y programas que existen destinados al colectivo de adultos y de adultos mayores. 


\section{LA OFERTA FORMATIVA REGLADA: EDUCACIÓN DE ADULTOS EN EL SISTEMA EDUCATIVO ESPAÑOL}

El Ministerio de Educación y Ciencia (MEC) apuesta por el fomento del aprendizaje a lo largo de la vida, para ello facilita el acceso a la educación contando con modalidades de formación presencial, a distancia, programas internacionales, formación docente relacionada con la educación permanente, premios y ayudas destinadas a la promoción de ésta, etc. En relación a la educación permanente, el Ministerio de Educación y Ciencia (2013) afirma en su Web (http://www.mecd.gob.es/portada-mecd/) que:

Todas las personas deben tener la posibilidad de formarse a lo largo de la vida, dentro y fuera del sistema educativo, con el fin de adquirir, actualizar, completar y ampliar sus capacidades, conocimientos, habilidades, aptitudes y competencias para su desarrollo personal y profesional.

La Ley Orgánica de Educación (LOE) de 2006, estructura y organiza el sistema educativo español en sus niveles no universitarios. Destacamos entre sus principales objetivos el propósito de fomentar el aprendizaje a lo largo de la vida. En este sentido señalamos el siguiente extracto del artículo 5.2. de dicha ley: "[...] facilitará a las personas adultas su incorporación a las distintas enseñanzas, favoreciendo la conciliación del aprendizaje con otras responsabilidades y actividades". Orientado en este sentido, el texto normativo regula, entre otras etapas educativas, la educación de adultos, indicando que la formación destinada a este colectivo puede ser reglada o no reglada, desarrollada en diversos contextos (laborales y/o socioculturales), dando respuesta a los siguientes objetivos:

a) Adquirir una formación básica, ampliar y renovar sus conocimientos, habilidades y destrezas de modo permanente y facilitar el acceso a las distintas enseñanzas del sistema educativo. b) Mejorar su cualificación profesional o adquirir una preparación para el ejercicio de otras profesiones.

c) Desarrollar sus capacidades personales, en los ámbitos expresivos, comunicativo, de relación interpersonal y de construcción del conocimiento.

d) Desarrollar su capacidad de participación en la vida social, cultural, política y económica y hacer efectivo su derecho a la ciudadanía democrática.

e) Desarrollar programas que corrijan los riesgos de exclusión social, especialmente de los sectores más desfavorecidos.

f) Responder adecuadamente a los desafíos que supone el envejecimiento progresivo de la población asegurando a las 
personas de mayor edad la oportunidad de incrementar y actualizar sus competencias.

g) Prever y resolver pacíficamente los conflictos personales, familiares y sociales. Fomentar la igualdad efectiva de derechos y oportunidades entre hombres y mujeres, así como analizar y valorar críticamente las desigualdades entre ellos.

El 17 de mayo el Consejo de Ministros aprobó la remisión a las Cortes Generales del Proyecto de Ley Orgánica para la Mejora de la Calidad Educativa (LOMCE), el cual recoge en su exposición de motivos que especifica que "La educación inicial es cada vez más determinante" de esta forma justifican que la educación se debe extender más allá del sistema educativo, así como se afirma la posibilidad de que el alumnado vaya transitando por diversos ámbitos educativos según su "vocación, esfuerzo y expectativas vitales" aportando respuesta a la necesidad de educación permanente. En el Artículo 68, relativo a las Enseñanzas obligatorias establecen los procedimientos de obtención de la titulación para el alumnado adulto especificando en su apartado 1 que: "Las personas adultas que quieran adquirir las competencias y los conocimientos correspondientes a la educación básica contarán con una oferta adaptada a sus condiciones y necesidades".

Los programas formativos desarrollados dentro del Sistema Educativo los podemos clasificar siguiendo la propuesta de MorenoCrespo (2011, pp. 304-307):

$\checkmark$ Educación Básica de Personas Adultas: Las Enseñanzas Iniciales comprenden la Alfabetización (Nivel I) y la consolidación de conocimientos y técnicas instrumentales (Nivel II). De igual forma, se incluye la enseñanza del español como lengua extranjera.

$\checkmark$ Enseñanzas de Educación Secundaria para Personas Adultas: Estas enseñanzas representan en Nivel III y son equivalentes a las Enseñanzas Obligatorias de Primaria y Secundaria en Régimen ordinario. Al finalizar este nivel se obtiene el título de Graduado en Educación Secundaria. Estas enseñanzas se podrán realizar tanto por la modalidad presencial como por la modalidad a distancia y estarán destinadas a las personas mayores de 18 años que no dispongan de una titulación básica.

$\checkmark$ Educación Secundaria de Personas Adultas a Distancia (ESPAD): El Centro para la Innovación y Desarrollo de la Educación a Distancia (CIDEAD) ${ }^{13}$, gestiona el ESPAD y posee la función de

13 Se encuentra integrado en el Centro Nacional de Información y Comunicación Educativa. Se trata de un Centro orientado a la producción, dirección y coordinación, tanto de recursos didácticos como de ordenación académica y que, en definitiva, ha de dar respuesta a las necesidades de aquellas personas que no pueden asistir a clase 
coordinar y organizar los elementos y procesos de la Educación a Distancia, así como facilitar el acceso a la educación de las personas adultas (mayores de 18 años) y, también, del alumnado en edad escolar que, por circunstancias personales, sociales, geográficas $u$ otras de carácter excepcional, se ven imposibilitados para seguir enseñanzas a través del régimen presencial ordinario (R.D. 1.180/1992 de 2 de octubre). En todo caso deberán acreditar los estudios requeridos para el acceso a estas enseñanzas (http://www.cidead.es).

Una vez superados los módulos que componen estas enseñanzas, el alumnado obtiene el título de Graduado en Educación Secundaria. Con este título se puede acceder a Ciclos Formativos de Grado Medio de Formación Profesional o al Bachillerato

Bachillerato a distancia y nocturno: El Centro para la Innovación y Desarrollo de la Educación a Distancia (CIDEAD) 14, gestiona las enseñanzas de Bachillerato a distancia, requiriendo como requisito de acceso el título de Graduado en Educación Secundaria o equivalente. Una vez que el alumnado accede al título de Bachillerato, tiene la opción de presentarse a las pruebas de Aptitud para Acceso a la Universidad o a un Ciclo Formativo de Grado Superior de Formación Profesional (http://www.cidead.es).

$\checkmark$ Formación profesional: Dentro de la formación orientada al trabajo encontramos la Formación Profesional Reglada que está destinada a los mayores de 16 años para la Formación Profesional de Grado Medio y a mayores de 18 años para la Formación Profesional de Grado Superior. La finalidad de la formación profesional es cualificar al alumnado para el desempeño de una profesión con el objetivo de la inserción en el mundo laboral (http://www.mec.es/educa/formacionprofesional y http://www.todofp.es).

$\checkmark$ Formación profesional a través de Internet: Se trata de un recurso ofrecido por el Ministerio de Educación que facilita el acceso a esta formación a aquellas personas que por tener otras ocupaciones o responsabilidades no disponen de las circunstancias propias requeridas para desarrollar estas enseñanzas de forma presencial. Los requisitos de acceso y las horas lectivas son similares a la modalidad presencial, se

\footnotetext{
y desean una formación académica; así como a los requerimientos educativos que plantea una sociedad en continua evolución, que considera la formación como una constante a lo largo de toda la vida.

14 Se encuentra integrado en el Centro Nacional de Información y Comunicación Educativa.
} 
desarrolla una evaluación continua, junto con evaluaciones finales presenciales. La superación de estas enseñanzas supone la obtención de un Ciclo Formativo de Grado Medio o un Ciclo Formativo de Grado Superior (http://www.mecd.gob.es/fponline/).

$\checkmark$ Programas de cualificación Procesional Inicial (PCPI)15: Orientados a mayores de 16 años (excepcionalmente mayores de 15 años) que no han obtenido el título de Graduado en Educación Secundaria Obligatoria, con el objeto de dotarles de una cualificación profesional básica para favorecer su inserción sociolaboral, sin perjuicio de continuar su formación dentro o fuera del sistema educativo.

$\checkmark$ Enseñanza oficial de idiomas: Desarrollado por el Ministerio de Educación y Ciencia con la participación de las Consejerías de Educación de las Comunidades Autónomas, Radio Televisión Española y la BBC. Comprende la oferta formativa que permite la obtención a distancia del Certificado Oficial del Ciclo Elemental del idioma Inglés.

\section{LA OFERTA EDUCATIVA FORMAL Y SOCIOCULTURAL}

El abanico educativo de posibilidades que se extiende ante nosotros es bastante extenso, por lo que nos centraremos en los programas formativos más destacables. Desde las Enseñanzas Profesionales podemos poner el acento en la Formación continua, que da respuesta al derecho de los trabajadores y trabajadoras a actualizar sus conocimientos profesionales y, por otro lado, la Formación profesional ocupacional, orientada a la inserción sociolaboral de personas en situación de desempleo y que se desarrolla en Casas de Oficios y Escuelas Taller para menores de 25 años y en Talleres de Empleo para quienes se encuentran en la misma situación pero se encuentran en la franja de edad de los 25 a la edad de jubilación ${ }^{16}$.

15 Los Programas de Garantía Social (PGS) que se encontraban fuera del sistema educativo (educación formal) con la LOGSE, han sido sustituidos por la LOE por Programas de Iniciación Profesional (PIP) que se sitúan dentro del sistema educativo reglado. Se trata de programas de formación para jóvenes que, habiendo cumplido al menos los 16 años, no alcancen los objetivos de la Educación Secundaria Obligatoria. La finalidad de estos programas es mejorar la formación general de este alumnado y capacitarles para realizar determinados oficios, trabajos y perfiles profesionales. Son enseñanzas no regladas cuya finalidad es facilitar la inserción laboral o la reinserción educativa de los jóvenes.

16 Actualmente en España se está produciendo un retraso en la edad de jubilación de los 65 a los 67 años. 
Un proyecto interesante y novedoso, aunque lleva funcionando desde hace más de diez años, es el Aula Mentor, diseñado como un sistema de educación a distancia desarrollado a través de Internet. El objetivo es seguir la docencia de cursos a través de un ordenador, conexión a Internet ${ }^{17}$ y el material de apoyo facilitado. El programa educativo está abierto para cualquier persona interesada con independencia de su titulación y nivel de estudios (http://www.mentor.educacion.es).

Por otro lado, en una línea similar, contamos con Radio Ecca, que desde su origen en 1965, se ha orientado al uso de la tecnología como herramienta pedagógica y ha adaptado sus enseñanzas a un nuevo modelo educativo. Las posibilidades de Radio ECCA son cada vez más amplias, más abiertas y más asequibles para cualquier persona, llegando a todos los niveles (http://www.radioecca.org).

Además contamos con los programas formativos desarrollados desde las Universidades Populares, que están orientados a proporcionar a todos los ciudadanos adultos y jóvenes la oportunidad de aprender activamente generando conciencia ciudadana y una actitud crítica ante las situaciones sociales que les rodean, así como el compromiso y responsabilización de sus propios derechos y deberes.

Compartiendo ideario y orientación, los Ateneos Libertarios pretenden la transformación radical de la sociedad, una transformación revolucionaria, a través del proceso de concienciación y compromiso cotidiano inmersos en la propia comunidad en la que se encuentran. Desde los ateneos libertarios se promueven los movimientos sociales, contemplando el acceso abierto para toda aquella persona que quiera participar. Se trata de cursos, proyectos, charlas, movilizaciones ciudadanas, exposiciones callejeras, coloquios, etc. Todas estas actividades se desarrollan en reuniones informales.

\subsection{Las Aulas de Mayores}

Visto el panorama de la Educación de Personas Adultas en España, nos centramos en el Programa Formativo que está siendo un éxito dentro y fuera de nuestras fronteras. Se ha extendido por todo el territorio español de Universidad en Universidad, abarcando asociaciones, obras sociales y fundaciones. Su éxito queda recogido en el Libro Blanco del Envejecimiento Activo editado por la Junta de Andalucía (2010) y el editado por el Gobierno de España (2011), sumado al reconocimiento que realiza la Consejería para la Igualdad y Bienestar Social de la Junta

\footnotetext{
17 El alumnado puede acudir a centros de referencia desde los cuales puede hacer uso de los ordenadores y de la conexión a Internet.
} 
de Andalucía en forma de galardón a las Aulas Universitarias de Formación Abierta para mayores de 55 años como Mejor Proyecto de Envejecimiento Activo (2011). De este modo se llevan desarrollando Programas Universitarios para Mayores desde los años 50 surgiendo aulas de formación, cursos de perfeccionamiento y otras actividades formativas.

Las Aulas de Mayores son una respuesta educativa de carácter formal y sociocultural ofertado principalmente desde las universidades. Con esta opción se contemplan los dos espacios de intervención educativa dentro de la sociedad destinados para las personas adultas mayores que Sáez Carreras (2003, p. 35) menciona en los siguientes términos: "[...] las Universidades, pensando en el sistema educativo formal, y luego el universo de los Servicios Sociales en la Comunidad, asociado más a una visión no formal de la Educación". En este sentido la Universidad cobra relevancia, tal y como deja patente Hernández Pina y Díaz Fernández (2010, p. 74) cuando destaca su papel para "[...] dar forma a la sociedad europea del conocimiento a través de su compromiso de amplia participación y educación permanente, así como mediante su promoción de la calidad y la excelencia en las actividades de docencia, aprendizaje, investigación e innovación".

El objetivo de las Aulas de Mayores es la apertura hacia las personas adultas mayores dando respuesta a la necesidad de realizar un aprendizaje a lo largo de toda la vida, con la finalidad de fomentar las relaciones intergeneracionales, romper el aislamiento social y favorecer su autonomía. La diversidad que encontramos en estas ofertas educativas, hace que nos centremos en las tres características comunes que comparten estos programas. En primer lugar encontramos la edad de acceso (para mayores de 50/55 años), requisitos previos (niveles básicos, si se exigen suelen ser competencias relativas a la lecto-escritura o incluso no son requeridos) y que la certificación otorgada al finalizar la formación no equivale a una titulación académica reglada; es decir, se trata de una certificación no competitiva (Cruz Díaz, Moreno-Crespo y Rebolledo Gámez, 2012a; Cruz Díaz, Rebolledo Gámez y Moreno-Crespo, 2012b; Moreno-Crespo, 2010; Moreno-Crespo, 2011; Moreno-Crespo y Cruz Díaz, 2012; Moreno-Crespo et al., 2009). En este caso, nos podemos cuestionar cuál es la finalidad de formarse para estas personas, ante lo cual, podemos tomar como respuesta la aportación de Madrid (1994, p. 107) al hablar de la educación de adultos y de las universidades populares:

[...] este proceso de enseñanza-aprendizaje no se dirige prioritariamente hacia la adquisición de determinados conocimientos teóricos, sino que se orienta más bien hacia el 
desarrollo de habilidades, capacidades, autoestima, hábitos, valores sociales de discusión, trabajo en grupo y participación en tareas colectivas, (...) que le permitan a este alumno adulto protagonizar su propio proceso de aprendizaje y ser al mismo tiempo creador de cultura (aprendizaje polivalente y participativo).

Habiendo despejado la filosofía de este tipo de programas, que cuenta con un marcado carácter sociocultural, alejado del interés por la compilación meramente academicista del conocimiento, destacamos que la metodología que en la que se basa es el autoaprendizaje. El espíritu de las Aulas de Mayores descansa sobre el "placer de saber". De esta forma, se permite la flexibilidad de profundización, por parte del alumnado, en aquellos saberes que son más de su interés. Se trata de un proceso de enseñanza-aprendizaje donde el itinerario se va consolidando en la medida que el alumnado avanza en los conocimientos, decidiendo en este quehacer la dirección del camino y la intensidad en la que se realizará. Por su puesto, con orientación y guía fundamentales de profesorado especialista. En este marco, la docencia bebe de forma importante de las experiencias de la propia persona participante, así como sus necesidades e intereses, conformando un consolidado aprendizaje significativo, más propicio y duradero en edades avanzadas (Cruz Díaz et al., 2012b; Moreno-Crespo, 2010; Moreno-Crespo, 2011; Moreno-Crespo y Cruz Díaz, 2012; Moreno-Crespo et al., 2009).

Queda claro que las ideas directrices de estos programas son el desarrollo de la cultura y la investigación, despertar inquietud por el conocimiento, responsabilizar a los estudiantes (autogestión y autoaprendizaje) y orientar el incremento de los saberes. Sáez Carreras (2003, p. 36) comenta al respecto que: "Es cierto que sus motivaciones mayoritarias no tienden a obtener un sueldo en términos vocacionales 0 profesionales [...], de ahí que prefieran "temas humanos y existenciales" más que programas orientados "hacia el conocimiento técnico y hacia una profesión futura".

Queremos resaltar la diversidad, flexibilidad y heterogeneidad que existe en este tipo de ofertas, tomando como ejemplo la provincia de Sevilla. En la capital y provincia, la Universidad de Sevilla oferta el "Aula de la Experiencia" (ampliar información en http://aulaexperiencia.us.es/), que cuenta con plan de estudios estructurado en cuatro años, adaptado al formato del Espacio Europeo de Educación Superior. Las materias se dividen en básicas, complementarias y optativas. El alumnado puede ser solo asistente o asistir y ser evaluado, optando cada modalidad a una certificación de asistencia o aprovechamiento respectivamente. Queremos resaltar que el estudiante que se matricula en primero se enfrenta a un plan de 
estudios estructurado para cuatro años. Por otro lado, la Universidad Pablo de Olavide, realiza una oferta para la provincia de Sevilla denominada "Aula Abierta de Mayores" (ampliar información en http://www.upo.es/aula-mayores/), que cuenta con un programa de tres cursos académicos, para los que cada año se diseña el plan de estudios. Desde la Secretaría Académica, con la colaboración de profesorado especialista, se elaboran ofertas de materias. Éstas son valoradas y seleccionadas en los municipios en donde se lleva a cabo el programa, de forma que atendiendo a la demanda, intereses y expectativas del alumnado se ajusta, en la medida de lo posible, a dicha oferta de universidad. En este caso, a diferencia del anterior, el alumnado que se matricula en primero se enfrenta a un plan de estudios que se irá ajustando en la medida en que vaya avanzando de curso. Para finalizar y como contrapunto, incluimos el Aula de Mayores de Aires Creativos y la Fundación Cajasol que se desarrolla en Sevilla capital (ampliar información en http://www.aulademayores.com/principal.php). Como distintivo principal, comentar que se trata de cursos anuales que se encuentran divididos en una oferta de talleres (pintura, restauración, modelados, dibujo al natural...), una oferta académica (historia, historia del arte, música, flamenco...), una oferta de idiomas (inglés, francés, alemán e Italiano), una oferta de actividades físicas (yoga, gimnasia, taichí, senderismo), una oferta recreativa (bailes de salón, sevillanas y palillos), una oferta de nuevas tecnologías (informática, fotografía digital y retoque fotográfico digital) y otras actividades como taller de memoria, inteligencia emocional, formación para la felicidad, etc.

En relación a los programas formativos para personas mayores, destacamos que actualmente no existe un referente legal, como tal, que regule este tipo de formación quedando en manos de instituciones públicas y privadas. No obstante, ante el éxito de este tipo de formación el Ministerio de Cultura regula los Programas Universitarios para Mayores como red de centros bajo su coordinación (BOE de 21 de junio de 1980). Igualmente, debemos señalar al respecto que éstos inciden en el derecho a la educación y la participación de personas adultas y personas adultas mayores, así como la promoción de su implicación como agentes activos de la comunidad, aspectos todos cubiertos por las Aulas de Mayores. Compartimos en este sentido las afirmaciones de Moreno-Crespo et al. $(2012$, p. 9) sobre estos programas socioeducativos, que indican:

[...], que con su aparente simplicidad ha enganchado a docentes y alumnos en un viaje por el conocimiento, el desarrollo de la persona en sus múltiples facetas, la educación a lo largo de la vida entendida en todas sus etapas y como erradicación de las desigualdades desde la perspectiva de democratizar el acceso a 
la educación, incidiendo igualmente en la promoción de la calidad de vida y el envejecimiento activo.

La educación de las personas adultas mayores debe tener en cuenta la satisfacción de sus necesidades. La CEA (Confederación de Educación de Adultos), que durante sus trabajos de investigación y consulta ha prestado oídos a las opiniones de los mayores, ha podido constatar que existe una diversidad de intereses, de expectativas, que debe ser conocida para poder programas adecuadamente. En relación a este discurso, destacamos las consecuciones que enumeran Prieto Jiménez y Moreno-Crespo (2009, p. 8) en relación a los Programas Universitarios para Mayores:

$\checkmark$ Ofrecer actividades socioeducativas partiendo de los intereses del alumnado.

$\checkmark$ Promover y favorecer la formación continua y permanente, para el desarrollo pleno de las personas adultas.

$\checkmark$ Aprovechar la riqueza cultural de los mayores para potenciar su autovaloración y autoestima.

$\checkmark$ Propiciar actividades artísticas y creativas que propicien el uso de su tiempo libre.

$\checkmark$ Facilitar el voluntariado y la ayuda mutua como factores favorecedores de la solidaridad y la convivencia.

$\checkmark$ Promover las relaciones intergeneracionales, facilitando así la tolerancia.

En este sentido, podemos establecer una serie de objetivos generales de los Programas Formativos para Adultos Mayores, aunque el carácter abierto y adaptativo de este tipo de programas despliega una gran diversidad en objetivos y contenidos:

$\checkmark$ Contribuir al desarrollo personal, nunca acabado, de los adultos mayores desde una propuesta científica y cultural.

$\checkmark$ Procurar un espacio natural para establecer nuevas amistades.

$\checkmark$ Promover la integración intergeneracional y remover estereotipos negativos acerca del envejecimiento y la juventud.

$\checkmark$ Que se produzca un enriquecimiento mutuo.

$\checkmark$ Abrir un nuevo campo a los investigadores.

Debemos mantener presente que estos programas se convierten en una alternativa cultural y formativa real para un sector de la sociedad, sin establecer límites de edad, socioculturales, económicos, educativos, etc., y respondiendo al criterio de formación a lo largo de toda la vida se da respuesta a los desafíos y peticiones que la sociedad necesita. En esta línea Amador (2010, cit. en Moreno-Crespo, 2011) indica que estos programas deben partir deben partir de los siguientes criterios: 
$\checkmark$ Adaptarse al ritmo de aprendizaje propio de los adultos mayores. De tal forma que el proceso formativo se adecue a su cadencia, para ello deben controlar el tiempo y desarrollo de las tareas. Debemos favorecer que el tiempo no los condicione en la búsqueda de sus motivaciones y necesidades, de tal forma que las acciones formativas adapten sus horario, fines y expectativas de acuerdo a los sujetos a los que van dirigidos.

$\checkmark$ Debe procurarse que los participantes sean coparticipes en su proceso de formación. En la medida que esta va a depender de su compromiso y de su esfuerzo. Con lo que se debe facilitar su participación en la organización conjunta, lo que les hará sentir fundamentales y necesarios para el colectivo y el proceso, lo que potencia su motivación.

$\checkmark$ Hay que partir de su experiencia formativa previa (competencias, habilidades).Toda nueva formación debe partir de los conocimientos previos, relacionándolos (aprendizaje significativo). Ello va a conllevar reafirmarlos en sus habilidades (llevan aprendiendo toda su vida y pueden seguir haciéndolo), estas exigencias y su papel social pueden ser fuentes de motivación. En ocasiones las nuevas experiencias deben producir resultados positivos con rapidez para fortalecer su confianza.

Igualmente debemos tener presente que los conocimientos previos pueden dar lugar a resistencias al nuevo aprendizaje; por ello hay que procurar que afloren en el proceso de enseñanza. Así como que cada alumno y alumna es una realidad ya configurada y diferente a sus compañeros.

Hay que tener en cuenta sus hábitos y estrategias de estudio. Debemos tener en cuenta que los adultos mayores buscan aprender porque les es útil en su propia vida: social, familiar, etc.; así como el que los cambios suelen ser motivantes para el aprendizaje., en la medida que les ayude a lograr los cambios que buscan. La aplicación de los conocimientos puede convertirse en motivadora para el aprendizaje.

$\checkmark$ Partir de sus intereses, necesidades y motivaciones. Debemos de partir de objetivos claros y al alcance de los mayores; facilitándoles, en la medida de lo posible, medios y métodos adecuados para lograrlos. En importante que alguno de los objetivos lo consigan en poco tiempo, ello reforzará su seguridad. Así mismo, deberían combinarse objetivos cognitivos con aprendizaje de habilidades y modificación de actitudes y hábitos. Atendiendo a este criterio, sería pertinente, que, en ocasiones, no nos olvidemos de técnicas de estudio que les 
ayuden a enfrentarse con las necesidades educativas de su proceso de enseñanza.

Queda patente que los Programas Universitarios para Mayores deben tener una estructura ágil que permita adaptarse a las necesidades e intereses que vayan apareciendo en cada una de las situaciones. Esta estructura debe tener presente el contexto, a las personas, a la Universidad, debiendo mantener una correspondencia y relación permanente con todos y cada una de los programas y actuaciones universitarias.

\section{CONCLUSIONES}

En los últimos años, hemos podido comprobar como las Aulas de Mayores han puesto en marcha diferentes programas formativos y académicos destinados a las personas mayores que han ido sufriendo una serie de modificaciones, adecuándose a las necesidades y preferencias de su alumnado, así como a las posibilidades de las propias instituciones.

Si bien todos y cada uno de estos programas poseen una esencia e idiosincrasia propia, hemos de decir que les mueve un último fin común, el de acercar la formación a la población más adulta, facilitándole el acceso a unos estudios de los que, en la mayoría de los casos, no pudieron disponer de ellos en su juventud.

Teniendo en cuenta lo expuesto, podemos afirmar que se da importancia a la función social de las Aulas de Mayores frente a otros fines. Se procura una organización de los contenidos con la finalidad de formar, transformar y dar respuesta a las necesidades e inquietudes del colectivo frente a la compilación de contenidos. La confección del currículo en estos casos es adaptado al alumnado. Esto lleva a que la flexibilidad hacia las demandas de este alumnado sea valorada positivamente.

Matizando las finalidades propias de cada institución, así como apreciando la meta última que pretenden alcanzar, observamos que son muchos de estos programas los que atienden a su vez a una vertiente más social.

Las características propias de las Aulas de Mayores las convierte en idóneas para impulsar proyectos de investigación en donde el adulto mayor pueda ser actor y director del proceso, dependiendo de la metodología empleada.

En este sentido y reconociendo la relevancia socioeducativa y sociocultural de estos programas ofertados a personas adultas mayores, 
Sarrate y Pérez de Guzmán (2005, p. 54) destacan una serie de propuestas orientadas a promocionar futuras líneas de actuación, de las que nos hacemos eco resumidamente: 1) Incluir la educación de personas adultas en todas las iniciativas y programas sociales; 2) Reconocer que el aprendizaje de las personas adultas es una inversión y no solamente una partida de consumo social; 3) Aumentar las reservas de capital humano, 4) Efectuar innovaciones curriculares; 5) Utilizar métodos pedagógicos apropiados; y 6) Establecer estrategias adecuadas.

\section{REFERENCIAS BIBLIOGRÁFICAS}

Aguaded, J. I. y Pérez Rodríguez, M. (2007). La educación en medios de comunicación como contexto educativo en un mundo globalizado. En J. Cabero (Coord.), Nuevas Tecnologías aplicadas a la Educación (63-75). Madrid: McGraw-Hill.

Cruz Díaz, M.R. (2013). Una asignatura pendiente de muchos adultos y mayores, matricularse en la universidad. Diálogos: Educación y formación de personas adultas (Barcelona), 3(75-76), 63-71.

Cruz Díaz, M.R.; Moreno-Crespo, P. y Rebolledo Gámez, T. (2012a). El alumnado universitario mayor ante los recursos tecnológicos y los medios de comunicación social: el caso del aula abierta de mayores. En G. Pérez Serrano (Coord.), Envejecimiento activo y solidaridad intergeneracional: claves para un envejecimiento activo. Madrid: UNED.

Cruz Díaz, M.R.; Rebolledo Gámez, T. y Moreno-Crespo, P. (2012b). Indicadores de evaluación de los programas universitarios para mayores: experiencia del "Aula Abierta de Mayores de la UPO". En D. Cobos; A. Jaén, E. López, A.H. Martín y L. Molina (Coords.), I Congreso Virtual Internacional sobre Innovación Pedagógica y Praxis Educativa INNOVAGOGÍA 2012. Sevilla: AFOE.

España (2006). Ley Orgánica de Educación. Boletín Oficial del Estado, 4 de mayo de 2006, n 106, pp. 17158- 17207.

García Mínguez, J. (2004). Educación en personas mayores. Ensayo de nuevos caminos. Madrid: Narcea.

Gobierno De España (2011). Libro blanco del envejecimiento activo. Madrid: Gobierno de España, Ministerio de Sanidad, Política Social e Igualdad.

Hernández Pina, F. y Díaz Martínez, E. (2010). La formación de doctores en el contexto del EEES. Una formación basada en competencias. Revista Fuentes (Sevilla), 10, 69-82.

Junta De Andalucía (2010). Libro blanco del envejecimiento activo. Sevilla: Junta de Andalucía, Consejería para la lgualdad y el Bienestar Social. 
Madrid Izquierdo, J.M. (1994). Las universidades populares y la educación de adultos. Pedagogía social: revista interuniversitaria (Sevilla), 9, 107-114.

Mayán Santos, J. (2001). Educación universitaria para personas mayores. En Fundación La Caixa Galicia (Ed.), Familia, juventud y nuestros mayores: la actitud proactiva (347-365). Coruña: Fundación Caixa Galicia.

Medina Ferrer, B.; Llorent García, V.J. y Llorent Bedmar, V. (2013). Evolución y concepto de la educación permanente en España. Revista de Ciencias Sociales (Maracaibo), Julio-Septiembre, 51 1-522.

Montoya Sáenz, J.M. (2002). La educación social en las personas mayores. En M.P. Lebrero Baena, J.M Montoya Sáenz y J.M Quintanta Cabanas (Coords.), Pedagogía Social. Madrid: UNED.

Moreno-Crespo, P. (2010). Mayores y formación: Aprendizaje y calidad de vida. En J.C. Gómez (Coord.), I Congreso Científico de Investigadores en Formación (281-283). Córdoba: Universidad de Córdoba.

Moreno-Crespo, P. (2011). Mayores y Formación. Aprendizaje y Calidad de Vida. Tesis Doctoral (no publicada). Sevilla: Universidad Pablo de Olavide.

Moreno-Crespo, P. y Cruz Díaz, M.R. (2012). Promoción de la calidad de vida a través de los programas socioeducativos para mayores: Universidad y mayores. En D. Cobos; A. Jaén, E. López, A.H. Martín y L. Molina (Coords.), I Congreso Virtual Internacional sobre Innovación Pedagógica y Praxis Educativa INNOVAGOGÍA 2012. Sevilla: AFOE.

Moreno-Crespo, P.; Pérez De Guzmán Puya, M.V. y Rodríguez Díez, J.L. (2012). Saber interpretar la calidad de vida y el envejecimiento activo. En I Simposium Internacional sobre envejecimiento activo y solidaridad intergeneracional (1-10). Madrid: UNED.

Moreno-Crespo, P. y Pérez Pérez I. (2014). Estereotipos sobre la jubilación en pretitulados universitarios: proyecto de innovación docente. REIRE: revista d'innovació i recerca en educación (Barcelona), 7 (2), 53-70.

Moreno-Crespo, P.; Prieto Jiménez, E. y Amador Muñoz, L. (2009). La necesidad de proyectos socioeducativos para mayores: el caso del aula abierta de mayores. Universidad Abierta: Revista de Estudios Superiores a Distancia (Ciudad Real), 29, 141-152.

Olmedo Moreno, E.M. (2013). Enfoques de aprendizaje de los estudiantes y metodología docente: Evolución hacia el nuevo sistema de formación e interacción propuesta en el EEES. Revista de investigación educativa, RIE (Murcia), 31 (2), 411-429.

Ortiz Colón, A. (2004). La formación básica de personas adultas en Andalucía desde la perspectiva del profesorado. Revista Fuentes (Sevilla), 6, 126-145. 
Palomares Ruiz, A. (2011). El modelo universitario y el uso de nuevas metodologías en la enseñanza, aprendizaje y evaluación. Revista de educación (Madrid), (355), 591-604.

Pérez Serrano, G. (2001). Aprender a lo largo de la vida. Desafío de la sociedad actual. Ágora Digital (Huelva), 1, 1-17.

Prieto Jiménez, E.; Moreno-Crespo, P.A. (2009). Diseñando programas para nuestros mayores: el caso del Aula Abierta de Mayores. En G. Pérez Serrano, J. García Gutiérrez y A. De Juanas Oliva (Eds.), V Jornadas de Calidad de Vida en Personas Mayores. Envejecimiento Activo y Participativo. Madrid: UNED.

Sáez Carreras, J. (2003). Investigación e Intervención Educativa con Personas Mayores. En J. Sáez Carreras (Coord.), Educación y aprendizaje en las personas mayores. Madrid: Dykinson.

Sanz Fernández, F. (2002). Perfil y funciones del educador/formador de personas adultas. En E. López-Barajas Zayas y M.L. Sarrate Capdevila (Coords.), La educación de personas adultas: reto de nuestro tiempo. Madrid: Dykinson.

Sarrate Capdevila, M.L. (2002). La educación de personas adultas, ámbito prioritario de la educación permanente. En E. López-Barajas Zayas y M.L. Sarrate Capdevila (Coords.), La educación de personas adultas: reto de nuestro tiempo. Madrid: Dykinson.

Sarrate Capdevila, M.L. y Pérez De Guzmán Puya, M.V. (2005). Educación de las personas adultas. Situación actual y propuestas de futuro. Revista de Educación (Madrid), 336 (enero-abril), 41-56.

\section{FUENTES ELECTRÓNICAS}

Aula Mentor. [Consultado el 16 de octubre de 2013], http://www.mentor.educacion.es/

Centro para la Innovación y Desarrollo de la Educación a Distancia. [Consultado el 16 de octubre de 2013], http://www.cidead.es/

Formación Profesional a través de Internet. [Consultado el 16 de octubre de 2013], http://www.mecd.gob.es/fponline/

Ministerio de Educación, Cultura y Deporte. [Consultado el 16 de octubre de 2013], http://www.mecd.gob.es/portada-mecd/

Radio Ecca. [Consultado el 26 de abril de 2011], http://www.radioecca.org/

TodoFP.Es. [Consultado el 16 de octubre de 2013], http://www.todofp.es/ 


\section{Sobre la autora:}

\section{Pilar Moreno-Crespo}

Doctora por la Universidad Pablo de Olavide con la tesis Mayores y Formación. Aprendizaje y Calidad de Vida (no publicada) (2011). Licenciada en Filosofía y Ciencias de la Educación en la Universidad de Sevilla (2001), Master en Dirección de Centros Sociales para Personas Mayores (Universidad Pablo de Olavide, 2005) y Master Universitario en Educación Social y Animación Sociocultural (Universidad de Sevilla, 2002). Profesora en el Departamento de Ciencias de la Educación de la Universidad de Extremadura (España). Implicada en labores técnicas-educativas en el Aula Abierta de Mayores de la Universidad Pablo de Olavide (2009-2014). Las líneas de investigación desarrolladas se centran en la educación de personas adultas y personas adultas mayores, así como educación permanente, envejecimiento activo y la calidad de vida. Ha participado en Congresos Internacionales orientados al envejecimiento activo y la calidad de vida, incluido el celebrado en 2010 en el que se presentó el Libro Blanco del Envejecimiento Activo, presentado por la Junta de Andalucía.

\section{Para citar este artículo}

Moreno-Crespo, P. (2015). Educación a lo largo de la vida: aula de mayores. Revista Fuentes, 17, Diciembre, pp. 113-133. [Fecha de consulta: $\mathrm{dd} / \mathrm{mm} / \mathrm{aa}$ ].

doi: doi: http://dx.doi.org/10.12795/revistafuentes.2015.i17.05. 